NASA/TM-2000-210032

I

\section{Sensitivity of a Wave Structure to Initial Conditions}

Walter M.B. Duval

Glenn Research Center, Cleveland, Ohio 
Since its founding, NASA has been dedicated to the advancement of aeronautics and space science. The NASA Scientific and Technical Information (STI) Program Office plays a key part in helping NASA maintain this important role.

The NASA STI Program Office is operated by Langley Research Center, the Lead Center for NASA's scientific and technical information. The NASA STI Program Office provides access to the NASA STI Database, the largest collection of aeronautical and space science STI in the world. The Program Office is also NASA's institutional mechanism for disseminating the results of its research and development activities. These results are published by NASA in the NASA STI Report Series, which includes the following report types:

- TECHNICAL PUBLICATION. Reports of completed research or a major significant phase of research that present the results of NASA programs and include extensive data or theoretical analysis. Includes compilations of significant scientific and technical data and information deemed to be of continuing reference value. NASA's counterpart of peerreviewed formal professional papers but has less stringent limitations on manuscript length and extent of graphic presentations.

- TECHNICAL MEMORANDUM. Scientific and technical findings that are preliminary or of specialized interest, e.g., quick release reports, working papers, and bibliographies that contain minimal annotation. Does not contain extensive analysis.

- CONTRACTOR REPORT. Scientific and technical findings by NASA-sponsored contractors and grantees.
- CONFERENCE PUBLICATION. Collected papers from scientific and technical conferences, symposia, seminars, or other meetings sponsored or cosponsored by NASA.

- SPECIAL PUBLICATION. Scientific, technical, or historical information from NASA programs, projects, and missions, often concerned with subjects having substantial public interest.

- TECHNICAL TRANSLATION. Englishlanguage translations of foreign scientific and technical material pertinent to NASA's mission.

Specialized services that complement the STI Program Office's diverse offerings include creating custom thesauri, building customized data bases, organizing and publishing research results ... even providing videos.

For more information about the NASA STI Program Office, see the following:

- Access the NASA STI Program Home Page at http://www.sti.nasa.gov

- E-mail your question via the Internet to help@sti.nasa.gov

- Fax your question to the NASA Access Help Desk at (301) 621-0134

- Telephone the NASA Access Help Desk at (301) 621-0390

- Write to: NASA Access Help Desk NASA Center for AeroSpace Information 7121 Standard Drive Hanover, MD 21076 
NASA/TM-2000-210032

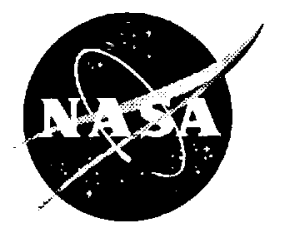

\section{Sensitivity of a Wave Structure to Initial Conditions}

Walter M.B. Duval

Glenn Research Center, Cleveland, Ohio

Prepared for the

38th Aerospace Sciences Meeting and Exhibit

sponsored by the American Institute of Aeronautics and Astronautics

Reno, Nevada, January 11-14, 2000

National Aeronautics and

Space Administration

Glenn Research Center 
Available from

NASA Center for Aerospace Information 7121 Standard Drive

Hanover, MD 21076

Price Code: A03
National Technical Information Service 5285 Port Royal Road Springfield, VA 22100 


\title{
SENSITIVITY OF A WAVE STRUCTURE TO INITIAL CONDITIONS
}

\author{
Walter M.B. Duval \\ National Aeronautics and Space Administration \\ Glenn Research Center \\ Cleveland, Ohio 44135
}

\begin{abstract}
Microgravity experiments aimed at quantifying effects of $\mathrm{g}$-jitter via controlled sinusoidal forcing transmitted on the interface between two miscible liquids have shown the evolution of a quasi-stationary four-mode wave structure oriented vertically. The sensitivity of the wave structure to phase angle variation is investigated computationally. We show that a slight variation of the phase angle is sufficient to cause a bifurcation to a two-mode structure. The dependence of phase angle on wave structure is attributed to sensitivity on initial conditions due to the strong nonlinearity of the coupled field equations for the parametric space of interest.
\end{abstract}

\section{Introduction}

Transport processes which are generated by buoyancy induced flows due to nonconservative body forces occur through an interface. In many practical problems such as crystal growth of binary alloys via directional solidification $^{1}$ or solution crystal growth ${ }^{2}$ the distribution of the concentration field near the interface determines the quality of the resulting crystal. This class of problem involves the coupling of the density field, through the body force, to the concentration and or temperature field. The availability of a low gravity environment, such as a Space Shuttle or the Intermational Space Station, offers a laboratory in which the body force is reduced to such an extent that mass transport occurring at an interface can potentially occur uniformly. However, random vibration or $\mathrm{g}$-jitter in that environment can affect mass transport so as to negate the advantage of the low body force. We study a model problem which considers the effect of controlled body forces to lead insight in understanding effects of g-jitter.

We consider the problem of two miscible liquids juxtaposed inside a cavity, with a sharp density interface initially, such that the body force is parallel to the interface. Experimental study to understand the effect of $\mathrm{g}$ jitter on such a system, using the Microgravity Vibration Isolation Mount (MIM) to input controlled vibration forces, ${ }^{3}$ shows that the interface can become unstable against Kelvin-Helmholtz instability and generate quasi- stationary waves with various modes. The effect of controlled vibration input to the system is to produce an oscillating shear flow at the interface. This problem has similarity to the classical unbounded parallel shear flow 4.5.6.7.8.9.10.11 in stably stratified fluids which generate Kelvin-Helmholtz instability. We investigate computationally the sensitivity of quasi-stationary $(q-s)$ waves on orientation of the oscillatory component of the body force through phase angle variation for the parametric space in which these structures were observed. We show that the observed two-mode $q-s$ waves are independent of phase angle for low Stokes-Reynolds $\left(R e_{s}\right.$ number. However, when the effect of nonlinearity becomes important for the four-mode $q-s$ waves, there exists narrow regions of phase angle for which the four-mode is stable.

In the following we investigate computationally the stability of two and four mode q-s waves when the initial condition on the phase angle is varied. We show the dynamics of the flow field which produces the q-s waves as well as the magnitude of the velocity field as a function of phase angle. For the parametric region in which nonlinearity becomes important we deduce the range of phase angle for which the four-mode q-s wave is stable.

\section{Model Problem}

Consider two miscible liquids introduced side by side inside a cavity as shown in Figure la. Such configuration is possible under a low gravity environment since the hydrostatic pressure jump can be made negligibly small due to the low steady component of the body force $\mathrm{n} g_{o}$. For certain parametric regions the interface can be stable and remain stationary in the vertical orientation. The effect of g-jitter may be studied systematically by introducing controlled vibration inputs $\mathrm{m} g_{o} \cos (\omega t+\theta)$ parallel to the interface. This can permit the study of the effect of amplitude $\left(\mathrm{m} \mathrm{g}_{o}\right)$, frequency $(\omega)$ and phase angle $(\theta)$ on the system. For the parametric range ( $\mathrm{ng}_{o}$ ) for which the interface is stable, one may investigate vibration inputs that cause instability. 
Given a stable interface oriented vertically inside a cavity separating two miscible liquids, the system will deviate from thermodynamic equilibrium; the interface will spread over time such that the system becomes homogeneous or uniformly mixed over a long time scale on the order of $\left(L^{2} \tilde{D}_{A B}\right)$. However depending on the body force field $g(t)$, for short time scales, the interface can remain stable or form various patterns according to the species continuity equation

$$
\frac{D C}{D t}=\tilde{D}_{A B} \nabla^{2} C
$$

The initial value of the two liquids may be prescribed as follows,

$$
t=0 \quad C(x, y, 0)= \begin{cases}1 & 0 \leq x<L / 2 \\ .5 & x=L / 2 \\ 0 & L / 2<x \leq L\end{cases}
$$

The fluid on the left and right hand side has respectively the value of 1 and 0 , and the interface has the value of 0.5 . The boundaries have a normal $(\vec{n})$ and the condition of impermeability is satisfied,

$$
\nabla C \cdot \vec{n}=0 \text { on } \Gamma \text {. }
$$

For parametric space in which the nonlinear convective terms in equation (1) are negligible, if the cavity domain is approximated to be infinite, a closed form solution shows

$$
C(x, t)=\frac{C o}{2} \operatorname{erfc} \frac{x}{2 \sqrt{\tilde{D}_{A B} t}}
$$

that the concentration field diffuses uniformly as a function of time. Whereas for parametric regions in which the interface becomes unstable, the nonlinear convective terms become dominant. This means that the characteristic of the flow field

$$
\vec{V}=\vec{V}(x, y, t ; \Lambda)
$$

dictates the pattern of the interface for a vector space of parameters $\Lambda$. Since the density field, which is a linear function of the concentration, field

$$
\rho=\bar{\rho}(1+\beta \Delta C)
$$

is coupled to the body force, the momentum equation

$$
\bar{\rho} \frac{D \vec{V}}{D t}=-\nabla p+\bar{\mu} \nabla^{2} \vec{V}+\rho \vec{g}(t)
$$

and the equation of incompressibility

$$
\nabla \cdot \vec{V}=0
$$

with no slip at the boundaries $(\Gamma)$

$$
\vec{V}=0 \text { on } \Gamma
$$

must be satisfied. The overbar denotes average values. The body force considered consists of a steady and oscillatory component of the form

$$
\vec{g}(t)=\mathrm{n} g_{o}+\mathrm{m} g_{o} \cos (\omega t+\theta)
$$

This class of problem represents an initial value problem, whose solution may depend sensitively on the initial conditions set by the prescribed density of the fluids or the nonconservative body force field prescribed by $\mathrm{n} g_{0}, \mathrm{~m} g_{e}$, and $\theta ; \theta$ may also be varied independently while keeping the other variables fixed to investigate the sensitivity to initial conditions.

Dimensionless parameters of the problem may be obtained by selecting the characteristic length, time, velocity, as

$$
L_{c}=\sqrt{\frac{\bar{v}}{\omega}}, T=\frac{1}{\omega} \text { and } U_{c}=\frac{\Delta \rho}{\bar{\rho}} \frac{m g_{o} L_{c}{ }^{2}}{\bar{v}} .
$$

The Stokes length scale was selected since the growth of waves is generated by the short wavelength instabilities, 12 and it also leads to a minimum set of dimensionless numbers to characterize the problem. When the steady component of the body force dominates, the geometric length scale such as the height (H) and length (L) becomes more important.

For computation the above set of equations are transformed to vorticity-stream function formulation by taking the curl of the momentum equation, let

$$
u=\frac{\partial \Psi}{\partial y} \quad \mathrm{v}=\frac{-\partial \Psi}{\partial x} \quad \zeta=\frac{\partial \mathrm{v}}{\partial x}-\frac{\partial u}{\partial y}
$$

The resulting set of equations become

$$
\begin{gathered}
\frac{\partial^{2} \Psi}{\partial x^{2}}+\frac{\partial^{2} \Psi}{\partial y^{2}}=-\zeta \\
\frac{\partial \zeta}{\partial t}+\operatorname{Re}_{s}\left(u \frac{\partial \zeta}{\partial x}+v \frac{\partial \zeta}{\partial y}\right)=\left[\frac{\partial^{2} \zeta}{\partial x^{2}}+\frac{\partial^{2} \zeta}{\partial y^{2}}\right)+ \\
(A R+\cos (t+\theta)) \frac{\partial C}{\partial x} \\
\frac{\partial C}{\partial t}+\operatorname{Re}_{s}\left(u \frac{\partial C}{\partial x}+v \frac{\partial C}{\partial y}\right)=\frac{1}{S c}\left(\frac{\partial^{2} C}{\partial x^{2}}+\frac{\partial^{2} C}{\partial y^{2}}\right)
\end{gathered}
$$

Since we are considering a cavity with aspect ratio 1 $(\mathrm{H}=\mathrm{L})$, the parametric space can be succintly represented as

$$
\Lambda=\Lambda\left(\operatorname{Re}_{s}, A R, S c, \theta\right)
$$

in which,

$$
\operatorname{Re}_{s}=\frac{\Delta \rho}{\bar{\rho}} \frac{m g_{o}}{\omega^{3 / 2} \overline{\mathrm{v}}^{1 / 2}} \quad, \quad A R=\frac{\mathrm{n}}{\mathrm{m}} \quad, \quad S c=\frac{\overline{\mathrm{v}}}{\tilde{D}_{A B}} .
$$


Res denotes a Stokes-Reynolds number, amplitude ratio AR, and Schmidt number Sc.

Equations 11-13 were discretized in space using finite difference techniques. The sharp discontinuity of the interface was resolved using the Flux Corrected Transport algorithm for computation. We used third order Adams-Bashforth for time discretization. All computations were carried out for a $90 \times 90$ grid size. We are interested in investigating the sensitivity of phase angle variation on the solution of the above equations for a parametric range of interest.

\section{Discussion and Computation Results}

\section{Parametric Range}

We consider the stability of two and four-mode quasi-stationary waves as a function of phase angle for two cases. The two-mode case corresponds to viscous fluid pairs consisting of $22 \%$ deuterium oxide $\left(\mathrm{D}_{2} \mathrm{O}\right)$ with de-ionized water $\left(\mathrm{H}_{2} \mathrm{O}\right)$ on the left hand side, and $100 \%$ de-ionized water on the right side. The kinematic viscosities are equal, $v=0.01 \mathrm{~cm}^{2} / \mathrm{sec}$, with a binary diffusion coefficient $\tilde{D}_{A B}=2 \times 10^{-5} \mathrm{~cm}^{2} / \mathrm{sec}$, and density ratio $\Delta \rho \bar{\rho}=0.022$. The body force has a steady component $\mathrm{n} g_{o}=1 \times 10^{-6}\left(g_{o}\right.$, Earth's gravitational acceleration $)$, and oscillatory component $\mathrm{m}=1.72 \times 10^{-4}$ with a frequency $\mathrm{f}=0.1 \mathrm{~Hz}$. The values of the dimensionless parameters are $\mathrm{Re}_{s}=2.4 \times 10^{-3}, A R=5.8 \times 10^{-3}, \mathrm{Sc}=500\left(1 / \mathrm{Sc}=2 \times 10^{-3}\right)$. The four-mode case consists of a dilute mixture of ethylene glycol (20\%) and water on the left side and $100 \%$ de-ionized water on the right, with a mean viscosity $\bar{v}=0.0108 \mathrm{~cm}^{2} / \mathrm{s}, \tilde{D}_{A B}=1 \times 10^{-5} \mathrm{~cm}^{2} / \mathrm{s}$, and $\Delta \rho \bar{\rho}=0.022$. The steady component of the body force is the same as above, however, $m=2 \times 10^{-2}$ or $20 \mathrm{mg}$ and $\mathrm{f}=1 \mathrm{~Hz}$. The dimensionless parameters are respectively, $\operatorname{Re}_{s}=0.3375$, $A R=5 \times 10^{-5}, \mathrm{Sc}=1079\left(1 / \mathrm{Sc}=9.3 \times 10^{-4}\right)$. The cavity size is of height and width $\mathrm{H}=\mathrm{L}=5 \mathrm{~cm}$, hence aspect ratio of 1. The phase angle was varied from 0 to $2 \pi$ for each case.

\section{Global Bifurcation of the Interface}

We first discuss the base state case for which the phase angle is zero. Under ideal microgravity conditions in which all disturbances are isolated, it is possible to obtain an initial condition as shown in Figure la. The interface band is represented by values of .1 to .9 so that the effect of mass diffusion can be shown as it evolves. Since the two fluids are miscible, the interface would spread over time while remaining stationary in the vertical orientation to satisfy the species continuity equation (1) for $\operatorname{Re}_{s}=0$ over a time scale $T=L^{2} / \tilde{D}_{A B}$ of order $O\left(5 \times 10^{4} \mathrm{sec}\right)$ for a $1 \mathrm{~cm}$ diffusion width. However, when energy is injected into the system through some controlled means, such as a MIM, the characteris- tic time for instability to grow is on the order of 100 $\mathrm{sec}$, depending on the parametric input. For excitation with a frequency of $0.1 \mathrm{~Hz}$, the interface becomes unstable against Kelvin- Helmholtz instability due to an oscillating parallel shear flow which results in a twomode quasi-stationary wave, Figure $1 \mathrm{~b}$. The oscillating shear flow is generated by the oscillatory component of the body force. The instability is largely due to the creation of vorticity at the interface as shown in the last term of equation (12). Note that since the amplitude ratio is small $\mathrm{AR}=0.0058$, the mean field is negligible as a result the interface remains stationary, otherwise it would tilt. The spreading or width $\mathrm{W}$ of the isoconcentrates which indicates mass diffusion equal to $\mathrm{W}=0.55$ $\mathrm{cm}$ is due vibration driven convection. For comparison, the width predicted for mass diffusion when convection is negligible from equation (4)

$$
\delta=2 \sqrt{\tilde{D}_{A B} t}
$$

is on the order of $0.28 \mathrm{~cm}$ which is approximately 0.5 times less than the convective case. The nonlinear convective terms in the concentration field equation (13) nearly balance the diffusion terms $\left(R e_{s}=0.0024 \approx 1 S c=0.002\right.$ ), thus one would expect mass diffusion to occur.

Increasing the effect of nonlinearity $\left(R e_{s}=0.3375\right)$ as seen from the vorticity equation (12), increases the number of modes to four, Figure 1c. The intensity of the flow field drives the instability. In this case the interface remains relatively sharp in comparison to the two-mode case. The concentration field equation (13) shows that the nonlinear convective terms are dominant over the mass diffusion term $\left(R e_{s}=0.3375 \gg 1 S c=9.3 \times 10^{-4}\right)$. This means that the interface can be deformed by the flow field for the time scale considered with minimum mass diffusion. The interface width $\mathrm{W}=0.22 \mathrm{~cm}$ is 1.58 times greater than the diffusion distance $\delta=0.126 \mathrm{~cm}$.

\section{Flow Field Dynamics as a function of Phase Angle}

\section{Two-mode Case}

The flow field dynamics, which yields the twomode structure, is shown in Figure 2. Various phase angles are shown which typifie trends for variation in the range of $0^{\circ}$ to $360^{\circ}$. As the phase angle is varied the wavelength

$$
\lambda=\lambda\left(\operatorname{Re}_{s}, A R, S c, \theta\right)
$$

of the q-s wave remain constant, independent of phase angle. The jump discontinuity of the density at the interface initially causes the interface to evolve like a vortex sheet. The tangential discontinuity of the velocity field causes the oblate shape of the streamlines. 
Diffusion of mass at the interface smears the discontinuity as the flow evolves. The two-mode structure is produced by a single vortex. When the phase angle changes to $90^{\circ}$ the effect of the steady component of the body force dominates which effectively changes the flow field to a rotational flow, Figure $2 \mathrm{~b}$. This can be seen from the vorticity equation, note that at $90^{\circ}$ the oscillatory component of the body force is zero initially, thus the steady component dominates. This example underscores the sensitivity to initial condition of the flow field. On the other hand, for a phase angle of $180^{\circ}$ which corresponds to the condition when the oscillatory component of the body force goes through a relative ninimum, Figure 2c, the single cell structure bifurcates to multi-cells. The flow field is weak, as indicated by the magnitude of the velocity field, thus there is no effect on the deformation of the interface. Even though the flow field is unstable with respect to phase angle variation, the interface deformation remains stable and independent of phase angle.

The magnitude of the velocity field as a function of phase angle is shown in Figure 3. The behavior of the flow field is not symmetric with respect to 180 , there is a decrease in the magnitude of the flow field for the phase angle range of $180^{\circ}-360^{\circ}$. The maximum magnitude of the velocity occurs at $90^{\circ}$ and $270^{\circ}$ while the minimum occurs at $0^{\circ}, 180^{\circ}$, and $360^{\circ}$. This example illustrates that for low $\operatorname{Re}_{s}(0.0024)$, even though there is a $200 \%$ variation in the magnitude of the velocity field, the wavelength of the interface remains constant independent of the phase angle.

\section{Dynamics of Flow Field for four-mode Structure}

The dynamics of the flow field that generates a four-mode quasi-stationary wave is shown in Figure 4 for a phase angle of zero degrees. Initially there is an oscillating parallel shear flow at the interface, Figure $4 a$, the tangential discontinuity of the velocity field is shown in the vector field plot. Unlike the unbounded parallel shear flows ${ }^{5}$ which generates travelling KelvinHelmholtz instability waves, the boundary in our case cancels out the wave motion and produces quasistationary wave patterns. The recirculation of the flow field due to impingement and reflection at the top and bottom boundaries create a region of concentrated vorticity, thus secondary cells are formed. The interface evolves like a vortex sheet due to discontinuity of the tangential velocity at the interface region, Figure $4 \mathrm{~b}$. A line vortex is created due to Kelvin-Helmholtz instability that results in undulation of the interface. The vortices grow as time increase, Figure $4 c$, two rows are formed similar to unbounded shear flows as shown by the vector field. The crests and troughs of the interface is due to co-rotating vortex pairs, as shown by the vector field, which forms homoclinic orbits. The singularity point between the vortex pairs serve as natural centers which stretch and rotate neighborhood portions of the interface into its undulatory shape.

\section{Stability of the four-mode Structure as a function of Phase Angle}

The effect of phase angle on the wavelength of the interface as well as the dynamics of the flow is shown in Figure 5. The basic four-mode structure as observed experimentally ${ }^{3}$ occurs for a phase angle of $0^{\circ}$, $175^{\circ}$, and $360^{\circ}$. Typical bifurcation of the four-mode structure is shown for $\theta=4^{\circ}, 90^{\circ}$, and $270^{\circ}$. Note that a slight change of phase angle of $4^{\circ}$ causes a transition to a two-mode structure. For phase angle in the range of $5^{\circ}-172^{\circ}$ the initial characteristic of the flow field changes from an oscillating parallel shear to a rotational flow with a single cell which has a stable elliptic point. However, the oscillatory component of the body force destabilizes the elliptic point to a homoclinic point. Thus, two co-rotating vortices are formed as shown by the streamlines and vector field in Figure $5 \mathrm{c}$ for $90^{\circ}$. The signature of the oscillatory parallel shear flow is preserved. The co-rotating vortices effectively stretch and deform the interface into its sinusoidal pattern. A clockwise rotation of the flow field results for phase angle in the range of $5^{\circ}-172^{\circ}$. Whereas, for phase angle in the range of $180^{\circ}-355^{\circ}$ a counterclockwise rotation of the flow occurs, Figure $5 \mathrm{~d}$.

The magnitude of the velocity field as a function of phase angle is shown in Figure 6. Unlike the two mode case in Figure 3, there is symmetry with respect to $180^{\circ}$. The maximum velocity occurs at $90^{\circ}$ and $270^{\circ}$, whereas the minimum occurs in the neighborhood of $173^{\circ}$ and $353^{\circ}$ respectively. The basic four-mode structure occurs within $3^{\circ}$ bands, for phase angle of $0^{\circ}-3^{\circ}$, $175^{\circ}-178^{\circ}$, and $357^{\circ}-360^{\circ}$. The regions of stability of the four-mode structure is represented by the average dimensionless wavelength $\lambda^{*}=(\lambda H)$ as a function of phase angle in Figure 7 . The narrow dip of the curve where the relative minimum occurs represents regions of decreasing wavelength or higher mode numbers. Overall the four-mode structure is sensitive to phase angle, it is stable over a narrow neighborhood region, near $0^{\circ}, 175^{\circ}$, and $360^{\circ}$. Within the bands of $0^{\circ}-5^{\circ}$, $175^{\circ}-180^{\circ}, 355^{\circ}-360^{\circ}$, shorter wavelengths occur. The trends show that for increasing Stokes-Reynolds number Res, the wavelength of the interface depends on phase angle. The effect of nonlinearity is to shift the wavelength of the interface to shorter and shorter wavelengths. The results show the importance of initial conditions on wave structure and the importance of aligning an experiment correctly with respect to the body force in a microgravity environment. 


\section{Conclusions}

The stability of two and four-mode quasistationary waves is investigated as a function of initial conditions for phase angle in the range of $0^{\circ}$ to $360^{\circ}$. Two-mode q-s waves which are formed at low StokesReynolds numbers $\left(\operatorname{Re}_{s}=0.0024\right)$ are stable with respect to phase angle variation even though the flow field is unstable; the wavelength is independent of phase angle. However, when nonlinearity becomes important for higher $\operatorname{Re}_{s}(0.3375)$, the four-mode wave bifurcates to a two-mode over most of the phase angle range. Only a narrow region exists for which the four-mode is stable. The wavelength is dependent on phase angle. This finding underscores the sensitivity to initial conditions of experiments performed for parametric spaces where nonlinearity becomes important.

\section{References}

(1) Chang, J.C., Brown, R.A., Radial segregation induced by natural convection and melt/solid interface shape in the vertical bridgman growth. J. of Crystal Growth, 63, 343-364, 1983.

2) Radcliffe, M. D., Steffer, J. E., Cook, E. L., Cotting, J. F., Miller, C. R., Drake, M. C., Schroeder, F. S., and Stevens, Jr., D., Organic crystals in low. earth orbit, J. of Crys. Growth, Vol. 92, 581-590, 1988 .

3) Duval, W.M.B., Tryggvason, B.V., "Effects of gjitter on interfacial dynamics of two miscible liquids:Application of MIM," NASA TM 209789, 2000.

4) Rosenhead, L., "The formation of vortices from a surface of discontinuity," Proceedings of the Royal Society, A Vol. 134, pp. 170-192, 1931.

5) Patnaik, P.C., Sherman, F.S., and Corcos, G.M., "A numerical simulation of Kelvin-Helmholtz waves of finite amplitude," J. Fluid Mech. , Vol. 73, part 2, 1976, pp. 215-240

6) Hooper, A.P., Boyd, W.G.C., "Shear-flow instability at the interface between two viscous fluids," J. Fluid Mech., Vol. 128, pp. 215-240, 1983.

7) Choi, W., Camassa, R., "Long internal waves of finite amplitude," Phys. Rev. Lett., Vol. 77, No. 9, 1996.

8) Moser, R.D., Rogers, M.M., "Mixing transition and the cascade to small scales in a plane mixing layer," Phys. Fluids A 3(5), pp. 1128-1134, 1991.

9) Corcos, G.M., and Sherman, F.S., "Vorticity concentration and the dynamics of unstable free shear layers," J. Fluid Mech., Vol. 73, part 2, pp 241$264,1976$.
10) Winant, C.D., Browand, F.K., "Vortex pairing: the mechanism of turbulent mixing-layer growth at moderate Reynolds number," J. Fluid Mech., Vol. 63, part 2, pp 237-255, 1974.

11) Kelly, R.E., "The stability of an unsteady KelvinHelmholt- flow," J. Fluid Mech., Vol. 22, part 3, pp 547-560, 1965.

12) Jacqmin, D, Duval, W.M.B., "Small scale instabilities caused by oscillations normal to viscous fluid-fluid interface," J. Fluid Mech., Vol 186, pp 495-511, 1988.

13) Jacqmin, D., Stability of an oscillated fluid with a uniform density gradient," J. Fluid Mech., Vol. 219, pp. 449-468, 1990. 
Public reporting burden for this collection of information is estimated to average 1 hour per response, including the time for reviewing instructions, searching existing data sources, gathering and maintaining the data needed, and completing and reviewing the colfection of informantion. Send comments regarding this burden estimate or any other aspect of this Davis Highway, Suite 1204, Arlington, VA 22202-4302, and to the Office of Management and Budget, Paperwork Reduction Project (0704-0188), Washington, DC 20503.
1. AGENCY USE ONLY (Leave blank)
2. REPORT DATE
May 2000
3. REPORT TYPE AND DATES COVERED
Technical Memorandum

4. TITLE AND SUBTITLE

5. FUNDING NUMBERS

Sensitivity of a Wave Structure to Initial Conditions

6. AUTHOR(S)

WU-101-53-00-00

Walter M.B. Duval

7. PERFORMING ORGANIZATION NAME(S) AND ADDRESS(ES)

National Aeronautics and Space Administration

John H. Glenn Research Center at Lewis Field

Cleveland, Ohio 44135-3191

8. PERFORMING ORGANIZATION REPORT NUMBER

$E-12229$

9. SPONSORING/MONITORING AGENCY NAME(S) AND ADDRESS(ES)

National Aeronautics and Space Administration

Washington, DC 20546-0001 AGENCY REPORT NUMBER

NASA TM-2000-210032

11. SUPPLEMENTARY NOTES

Prepared for the 38th Aerospace Sciences Meeting and Exhibit sponsored by the American Institute of Aeronautics and Astronautics, Reno, Nevada, January 11-14, 2000. Responsible person, Walter M.B. Duval, organization code 6712, (2I6) 433-5023.

12a. DISTRIBUTION/AVAILABILITY STATEMENT

12b. DISTRIBUTION CODE

Unclassified - Unlimited

Subject Categories: 29, 34 and 64

Distribution: Nonstandard

This publication is available from the NASA Center for AeroSpace Information, (301) 621-0390.

13. ABSTRACT (Maximum 200 words)

Microgravity experiments aimed at quantifying effects of g-jitter via controlled sinusoidal forcing transmitted on the interface between two miscible liquids have shown the evolution of a quasi-stationary four-mode wave structure oriented vertically. The sensitivity of the wave structure to phase angle variation is investigated computationally. We show that a slight variation of the phase angle is sufficient to cause a bifurcation to a two-mode structure. The dependence of phase angle on wave structure is attributed to sensitivity on initial conditions due to the strong nonlinearity of the coupled field equations for the parametric space of interest.

\begin{tabular}{|c|c|c|c|}
\hline \multirow{2}{*}{\multicolumn{3}{|c|}{$\begin{array}{l}\text { 14. SUBJECT TERMS } \\
\text { Miscible liquids; Wave structure; g-jitter; Phase angle; Microgravity }\end{array}$}} & \multirow{2}{*}{$\begin{array}{l}\text { 15. NUMBER OF PAGES } \\
\frac{13}{\text { 16. PRICE CODE }} \\
\text { AO } 3 \\
\text { 20. LIMITATION OF ABSTRACT }\end{array}$} \\
\hline & & & \\
\hline Unclassified & Unclassified & Unclassified & \\
\hline
\end{tabular}

